\title{
On the Way to Dominating Cognition
}

\author{
Edward M. Pogossian \\ Institute for Informatics and Automation Problems of NASRA \\ e-mail:epogossi@aua.am
}

\begin{abstract}
Humans become powered enough to question the further types of their being in the universe but have not answers yet whether the solutions are in the corrections of their genomes, discovering of new types of organizations of humans or in transition to a new type of descends, humanoid machines or others.

Assuming that scenarios with dominating cognition are the most promising to resolve the challenges of evolvement of humans and are the most expected for the next stage of their being, while the kernel of effective cognition is universal for all types of further being of humans, we present constructive models of the kernel, question the completeness of representations and ways of its further study.

Keywords: Mental systems, Adequate, Constructive modeling, Cognition,Negentropics.
\end{abstract}

\section{Introduction}

1.1. 1. Following the cosmological views [33], the vast majority of realities we classify as entropics while interpreting Schrodinger [6] we classify as negentropics or negs, an island of not entropic realities $r$ that can gain energy from others to preserve in space and time certain roots, i.e., realities, say, constituents or doings of r, determining the identity of $r$.

Roots of negs, as it succeeds from definitions, have to include, at least, means to gain energy and ones to preserve those means that, in turn, require means to represent and affect realities, i.e., sensors and effectors, then, means to classify utilities, damagers and uncertains, i.e., classifiers of favorable, damaging or uncertain yet with respect to (wrt) the roots realities, as well as controllers ofthe doings.

1.2.1. We classify cellular realities, cellulars, as a type of negentropics that include alive cells in the roots able to reproduce and compose themselves into the organism while classifying humans as a type of cellulars.

1.2.1. Mental doings of humans are backing the root and induced goals. They are either genomic or cognized in the life time while cognized mainly by acquisition from the cultures of communities. 
Mental doings are represented by mental doers, mdoers, while mental classifying by mdoers or systems of mdoers, msystems (mss).

Mdoers and mss, have, we assume, at least, IDs and can be processed consciously, particularly, for explaining and understanding doings/doers in communications.

1.2.2. Mdoers include algorithms and are based, we assume, on and do over IDs of sensors, elaborate instructions for the effectors and are governed by the controllers.

Mss induce systemic classifiers, say, Factories, Computers, Chess Positions.

Mss, particularly, represent the goals, classify coexistence of nominated utilities/goals, i.e., represent relationships between them, can be processed to learn new utilities, to enhance effectiveness of mss or communicate them.

1.2.3. Acquisition and accumulation of mss, as well as revelation of mss, particularly, by learning new utilities or by enhancement of effectiveness of ad hoc mss, comprise the balk of doings of a type of mss, cognizers.

Processing of mss is governed, we assume, by controllers.

For example, controllers explain mss "Humans" of the author by resolving it into this, ongoing text, namely, by corresponding English words to IDs of constituents of the mss.

That resolution, in general, could be started from any constituents of target mss, selected constituents, thus their IDs could be chained in a variety of modes and details depending, particularly, on the intensions of the author.

1.2.4. A mighty way of enhancement of effectiveness of mss, and thus, cognizers, is the regularization of classifiers induced by mdoers and mss [47].

Namely, classifiers $\mathrm{Cl}$ of members $\mathrm{x}$ of communities $\mathrm{C}$ are regularized in $\mathrm{C}$ if accompanied by ontological in $\mathrm{C}$ methods, instructions allowing $\mathrm{x}$ regularly provide positive samples of inputs of $\mathrm{Cl}$, as well as let the members of $\mathrm{C}$ do the same by communicating with $\mathrm{x}$.

In constructive regularization, those samples can be provided deterministically and without any involvement of cellulars while, otherwise, they can be grown up from a priory given prototypes like cells or crystals, be the products of services to humans or machines.

1.3. Accelerated mental power approaches humans to "Homo Deus" [34] with a chance of reliable preservation of cellular roots while, in parallel, to entire crashing of cellulars on the earth.

The kernel of that strength is in the effectiveness of organizations of humans while the weakness is caused by the imperfectness of those organizations and their genomic heritage.

Humans become powered enough to question the further types of their negentropic being in the universe but have not answers yet whether the solutions are in the corrections of their genomes, discovering of new types of organizations of humans or in transition to a new type of descends, humanoid machines or others.

It is worth reminding that nowadays autonomous agents, a type of constructed negentropics, are challenging the cellular being of humans at all, as well as recall that following the Buddhist and Indian sources, humans being lemuroids or Atlantis in the far past could have not only cellular types of negentropicity [35].

1.4. 1. In what follows, first, we present the modified specification of the models of mss, mentals, introduced in [47], refine systemic classifiers and constructive regularization of classifiers.

We continue questioning the ways of proving that mentals can be adequate constructive models of mss. In addition to the approach in [47], based on examining performances of particular mss with the corresponded them mentals, we analyze the ways to achieve consistency of performances of structural models of connectivity neuron nets, for example, artificial neuron nets, with purely functional models of mss, mentals.

Assuming that the kernel of effective cognition is universal for all types of negs, thescenarios with dominating cognition are the most promising to resolve the challenges of evolvement of 
humans and are the most expected for the next stage of their being, finally, we question the completeness of representation of the kernel and ways of its further study.

1.5. Our models are based on and try to fuse findings of many outstanding researchers.

We refer to some of their publications [1-35] to study them in depth, as well as refer to some works [36]-[42], which can add to understanding of our ideas and their approbations [43]-[48].

\section{Constructed Mental Systems}

2.1.1. Doers, in general, are, we assume, realities having in- out- put parts and for available inrealities, i.e., realities at the input parts, either elaborate certain output realities or stay passive. In- out- realities comprise their in- out-domains, or in- out-doms.

Indoms wrt outputs are split into classes of equality, particularly, class of uncertainty (?) if inrealities don't cause outputs.

2.1.2. Doers are do-classifiersCl if indoms are split into two classes $+\mathrm{Cl}$ and ?Cl; otherwise they are corresponders, cors.

Apparently, identifiers of do-classifiers $\mathrm{Cl}$ by themselves are sufficient to indicate their classes of equality, i.e., the positives $+\mathrm{Cl}$, while classes of cors can be indicated by pairing those identifiers with corresponding outputs.

2.1.3. Doers of type of classifiers are sensors if inrealities are not necessarily pre-classified, of type of cors are effectors if inrealities are necessarily classified while are controllers if both inout- realities are necessarily classified.

2.2.1. Realities $\mathrm{I}\{\mathrm{i}\}$ are identifiers, IDs, of realities $\mathrm{R}\{\mathrm{r}\}$ and $\mathrm{Z}\{\mathrm{z}\}$ wrt $\mathrm{Z}$ if

- to any r,z unique IDs i(r), i(z) are corresponded

- to any r,z certain classifiers are linked allowing by IDs i to recall the corresponding $r$ or $z$

- any r can address any $\mathrm{z}$ to recall any $\mathrm{r}, \mathrm{z}$.

Identified realities of given $\mathrm{R}, \mathrm{Z}$ paired with their IDs are named nominals wrt Z.

$\mathrm{Z}$ and $\mathrm{R}$ can coincide for, say, $\mathrm{R}$ presenting members of communities or their mdoers.

Controllers Cns, are assumed, can assign IDs to given mdoers aimed to control their processing and in- out- interactions with realities.

Realities of $\mathrm{Z}$ can be interpreted as sets of Cns controlling in certain ways realities of $\mathrm{R}$ analogically to servers of "star" types controlling networks of computers or, seemingly, analogically to unicellular controllers.

2.2.2. Nominals wrt Cns where realities of $\mathrm{R}$ are outputs of doers, particularly, sensors, controllers or effectors, are named otids while sets of otids of doers d are the alphabets of $d$.

And sets of otids comprised from only some representatives of alphabets A1,A2, ..,An of doers $\mathrm{d} 1, \mathrm{~d} 2, \ldots, \mathrm{dn}$ are words in $A 1, A 2, \ldots, A n$.

2.2.3. Classifiers of $n$-tuples of nominals are $n$-place relationships named rels for $\mathrm{n}=2$.

Rels $(a, b)$ can be depended or not on the orders of their arguments.

2.3.1. Systems $H$ over nominals Nls containing rels Rls, i.e., Rls $<$ Nls, or systems $H$ over Nls/Rls,

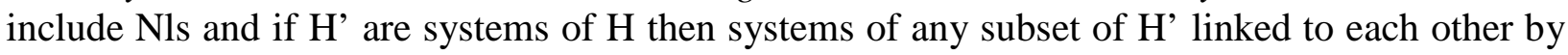
rels of Rls and nominated by IDs consistent with nominations of Nls are systems of $\mathrm{H}$ as well.

2.3.2. The totality $\mathrm{H}$ of systems over Nls/Rls comprise Nls/Rls nets where nodes a, b are IDs corresponded to systems of $\mathrm{H}$, the edges ( $\mathrm{a}, \mathrm{b}$ ) are corresponded to rels between the systems represented by nodes a,b , signed, "colored " by IDs of those rels and oriented from b to a if correspond to rels(a,b).

Nls/Rls nets are, in fact, colored and oriented nets where nodes $a$ depend on nodes $b$ if rels $(a, b)$ are corresponded to the edges linking $a$ and $b$. 
Assuming Nls are 1st layer systems of Nls/Rls nets, the systems of $n+1$ - layers are formed as systems over nominals of n-th layers and rels Rls.

2.4.1.Given controllers Cns, effectors Efs and sensors Sns nominated wrt to Cns, or ces, doers $D$ over IDs of ces, or doins or cesdoins D, are doers D nominated wrt to Cns while indoms of D, Ffs, Cns are words in alphabets of the outputs of D, Cns, Sns.

2.4.2. Bundles of otids of Sns,Cns and cesdoins at time $t$ are $t$ prints comprised into certain stores Pns and nominated wrt to Cns.

2.4.3.Basic cesnominals, or cesbns, include cesdoins D united with Cns, Efs,Sns,Pns nominated wrtCns.

2. 4.4. Systems of cesbnominals, or scesbns, are systems over Nls, Rls where Nls are cesbns.

The totalities of scesbns comprise cesnets Nts.

2.5. The following types of scesbns can equally correspond to algorithms, say in Markov, Java or other equal modes.

Equal to rules by Markov are types of doins, regularities, or regs, corresponding certain otids to only some selected words of indoms.

Algorithms are scesbns comprised from regs by rels similar to ones comprising rules into algorithms by Markov [29,30]. rules.

Cessbns algorithms, in fact, detail ones by Markov with respect to detailing the origin of

Scesbns of the types of "abstract classes", referring, say, to ones in Java, are systems of algorithms, or methods, in rels of the types: "attributed", "parented” and "done by", with other abstract classes eventually, comprising algorithms by Markov.

Abstracts expand abstract classes by allowing arbitrary Rls rels with other abstracts.

Finally, packages of abstracts and their libraries are mimicking the ones in Java.

2.6.1. While scesbns can range from the sets of disjoined to the totally connected to each other systems we refine mental systems, mss, as those of scesbns that are connectivity subnets $G$ of cesNts rooted in the nodes $a$ of Nts.

Namely, total connectivity scesbns $G$ rooted at nodes a n (or total a connectivity scesbns G, or total connectivity a/ scesbns $G$ )of cesNts are connectivity subnets of Nts rooted in a.

And a rooted scesbns $G$ ' of total connectivity a scesbns G, or a mentals, or, generally, mentals, are $a$ rooted connectivity subsystems of $\mathrm{G}$. Then, $a 1, a 2, .$. , an aspects of $G$ ' are a1,a2,.., an rooted connectivity subsystems of G'.

Apparently, connectivity a scesbns are a mentals and nodes a1,a2,.., an by themselves can be the aspects of G'.

The totalities of mentals of cesNts comprise cesthesauruses cesTh.

2.6.2.Decompositions of 1st depth, or 1st decompositions, of a mentals $G$ having nodes $a$ at some layer $k$ of cesNts are a1,a2,.., an rooted mentals of all subsystems G1,G2,..,Gn of $G$ with nodes $\mathrm{a} 1, \mathrm{a} 2, \ldots$, an of k-1 layers of Nts connected to a.

And if $\mathrm{G} 1, . ., \mathrm{G}$ are $\mathrm{i}$-th decomposition of $\mathrm{G}$ then $i-1$ th decomposition of $G$ will be the union of 1st decompositions of G1,..,Gn.

Apparently, the terminal decompositions of $G$ will be comprised from bnominals of Nts. The unions of $i$-th decompositions of $\mathrm{G}$ for $\mathrm{i}=1, \ldots, \mathrm{k}-1$ comprise total decompositions of $\mathrm{G}$.

2.6.3. Analogously can be defined j-th abstractions and total abstractions of a mentals $G$ in the way that 1 st abstractions of a mentals $G$ with nodes a at some layer $k$ of cesnets Nts could be $\mathrm{a} 1, \mathrm{a} 2, . .$, an rooted mentals $\mathrm{G} 1, \mathrm{G} 2, \ldots, \mathrm{Gn}$ of all subsystems of $\mathrm{G}$ with a1,a2,.., an at the $\mathrm{k}+1$ layers of Nts and connected to a, etc.

2.7.1.Thesauruses cesTh are assumed to be stored by analogy with storing libraries, say in Java. Namely, nodes of cesnets are stored with IDs of the nodes, the classifiers of IDs of the nodes, IDs of rels of nodes a with nodes $b$ along with IDs of those $b$. 
Nodes a corresponded to ces abstracts d, in addition, contain either the decision makers of $\mathrm{d}$ or the references to them.

2.7.2. Apparently, nodes corresponded to ces abstracts of cesnets or in cesTh will coincide with abstract classes of Java in the case when their rels with other nodes of cmnets are restricted by "attributed", "parented" and "done by" ones.

2.7.3. Started fr identifying common for communities $\mathrm{C}$ doers, mdoers and msystems, so far, we have specified doins, scesbns, cesnets and cesTh thesauruses as well as total connectivity scesbns, their connectivity subsystems, mentals, and the aspects of mentals representing, we assume, mss and the aspects of mss.

To question whether mentals can be adequate constructive models for mss and for that aim refine constructive modeling and adequacy as it follows.

\section{SYSTEMIC vs. DO CLASSIFIERS}

3.1. Classifiers in their min mode identify some realities while if they are regularized, they can regularly provide samples of their indoms. In turn, regularized classifiers can be models or adequate models of each other either constructive or not, as it follows.

3.2.1. Do classifiers $\mathrm{Cl}$ were defined as a type of doers that for realities at the input parts either elaborate certain outputs or stay passive, thus, splitting indoms into two classes +Cland?Cl, We assume that not only doers but any mss $\mathrm{h}$ induce certain systemic classifiers hsCl with positives +hsCl that can be presented by mentals corresponded to mss as follows.

3.2.2.1. Realities $r, r^{\prime}$ are (fuzzy) equal with respect to doins $d$ if $d$ is applied to $r, r^{\prime}$ outputs (fuzzy) the same otids. In other words, $d$ analyzing $r, r$ ' by their embedded regs doesn't find any (fuzzy) distinction between $r$ and r'.

Due to equality of realities, as a rule, they can be incomplete, approximate or fuzzy later on in refining equality we skip to name the option of their fuzziness.

3.2.2.2. Doers are equal if for any inputs their outputs coincide.

For constructiveness of that requirement, we assume that indoms of doers are made finite by some criteria.

Thus, doers are equal if their performances, i.e., the pairs input/output, for inputs of their indoms coincide.

3.2.2.3. Mentals $G, G$ ' are equal if certain doers d determine that decompositions of G,G'untill terminal doins are isomorphic wrt equality of corresponded to each other doins while those doins are linked by the equal rels.

3.3.Realities $r$ match to mentals $h$ if certain doers $d$ can reveal in $r$ equal to $h$ systems $h$ '. Thus, pairs (mentals h, d) determine systemic classifiers hsCl with positives $+\mathrm{hsCl}$.

\section{Regularized and Modeled Classifiers}

4.1.1. Classifiers $\mathrm{Cl}$ of members $\mathrm{x}$ of communities $\mathrm{C}, \mathrm{x} € \mathrm{C}$, are regularized if $\mathrm{x}$ can accompany $\mathrm{Cl}$, first, by methods Clrgz letting $\mathrm{x}$ provide positives of $+\mathrm{Cl}$ regularly, and second, $\mathrm{x}$ by ontological in $\mathrm{C}$ communicativesClcms can explain Clrgz to any $\mathrm{y} € \mathrm{C}$ why $\mathrm{y}$ can provide positives of $+\mathrm{Cl}$.

Regularized are, for example, classifiers of produced goods, grown up domestic plants and animals, services and skills provided hand to hand.

Scientists $\mathrm{x}$ discovered some realities aand classified them by regularized classifiers $\mathrm{Cl}$ can by Clrgz provide samples of a and by Clcms successfully explain to others how to do the same. 
3.4.2.Classifiers $\mathrm{Cl}$ are fuzzy regularized if they are regularized but to the extent that outputs of Clrgz only fuzzy match to $+\mathrm{Cl}$ and the fuzziness can range up to not matching to $+\mathrm{Cl}$.

4.1.2. Let's recall that positives of $+\mathrm{Cl}$ are realities ofindoms of do classifiers doCland are systems of doers for systemic classifiers sCl.

Particularly, if mss $\mathrm{h}$ are some do classifiers doCl positives matching thsCl will be doClthemselveswhile positives of $+\mathrm{doCl}$ will be some realities of indoms of $+\mathrm{doCl}$.

4.1.3. Corollary1. Regularized mss h are equal to hrgz methods wrt out realities of hrgz, i.e., if realities rare reproducible by hrgz then they match $h$.

Apparently, regularized mss $\mathrm{h}$ necessarily have to include systemic classifiers hsCL either explicitly or implicitly.

Statements on mss, as a rule, have also transparent fuzzy interpretations why often can be skipped later.

4.2.1.The samples of classified realities $\mathrm{r}$ can be constructed deterministically and totally independent from cellulars in constructive regularization, conrgz, or can be regularized not constructively, particularly be provided not deterministically, be grown up from a priory given realities like cells or crystals, be a product of services to humans or machines.

For example, mss Goods (Gds) representing producible in the frame of some civilization goods, saymss Computers (Cps) since are reproducible from totally not cellular realities.

Conrgz are mss Algorithms (Ags), represented either as Turing Machines, Post Productions, Markov algorithms or Recursive Functions that can be specified to be assembled from not cellular units by mathematicians or programmers, and even more, they are enumerable.

Mss Wheat, Domestic Animals are fuzzy regularized and grown up while mss Services of cellulars , say Treatments by Doctors, are fuzzy regularized and are inseparable from cellulars.

Assuming a numerical scale for fuzziness of regularization its variable $f$ might be zero for Cps, Gds and Ags, range from zero for mss Deterministic Methods (DM) to $1 / 2$ for the Heuristics, and have $\mathrm{f}=1$ for mss Conscious, Emotions, Passions questioned yet to be regularized.

4.2.2. Humans tend to regularized classifiers $\mathrm{Cl}^{`}$ for the advantage not only passively classify but actively provide positives of classifiers.

Constructive regularization let, in addition, exempt positives from being cellulars, thus, expanding leverages to amplify target doings . Recall, for example, transition from riding by horses to cars or trains.

4.3.1. Regularized mss $h$ are modeling mss $h *$ if out realities of hrgz match $h^{*}$.

Regularized mss $h$ are adequately modeling mss $h^{*}$ if they are modeling $h^{*}$ and for any realities $\mathrm{r}^{*}$ matching $\mathrm{h} *$ hrgz can produce realities $\mathrm{r}$ equal to $\mathrm{r}$ *

Apparently, regularized mss $\mathrm{h}$ are adequately modeling themselves.

4.3.2.By Church DM can be adequately modeled by Ags, i.e., to any DM equal Ags can be corresponded.

Recalling Corollary1, it can be stated the

Corollary2. Regularized mss h are equal to certain algorithms wrt out realities matching h.

4.3.3. Church thesis, we assume, can be expanded for fuzzy and not deterministic algorithms and methods as well.

Namely, DM can be expanded to Methods (Mds) and Ags to fAgs adding to Ags, say, probabilistic, distributed and heuristic methods / algorithms.

Corollary3. Regularized mss h (fuzzy or not) are equal to certain algorithms (fuzzy or not) wrt realities matching $h$. 


\section{Consisting Functional and Connectivity Mental Models}

\subsection{Adequacy of mss is questioned functionally and connectively.}

Functional questioning examines the equality of performances of mss and models of mss of any origin.

In contrast, in connectivity modeling it is required that the units of the models mss have to be adequate models of the units of nerves systems, neurons, and, particularly, looking for adequacy of mss with artificial nets of neurons, ANN.

Following the ideas of functional modeling, we examine our models of mss, mentals, by consistency of their performances with targeted mss, first of all cognitive ones, providing models of those mss aimed to support our expectations, or the hypothesis Ham, that mentals might be adequate models of mss [47]..

So far, we have advanced in supporting Ham, particularly, by the models of representations and inductive learning [36,40-42], utilization of realities by acquisition or by strategies [38, 39,], matching to classifiers [44-46] and communications [47].

5.2.1. The hypothesis Ham, apparently, induce the question to be studied, namely, whether thesauruses cesTh, i.e., thetotalities of mentals defined in [47]and above, nervy nets NN are equal wrt to representation and performances of mss.

5.2.2. These days adequacy of connectivity models of $\mathrm{NN}$ are intensively examined, specially, for artificial neuron nets, ANN, that are consistent with a variety of physiological classifiers of NN.

Actually, the question arises about the hypothesis eANN?cesTh on equality of ANN and the nets of mentals, cesTh, or in other words, whether ANN can be organized in the way to be equal to NM with respect of representation and performance of mss?

Our expectations for the positive answer to eANN?NM are supported by the following premises. 5.3.1. Doers of cesTh, doins, and doers of ANN, neurons, both are doers over IDs of nominals over given sensors/effectors/controllers.

5.3.2. Nets of neurons and their constituent neurons are classifiers either of types of sensors or of classifiers of IDs of nominals or $n$ tuples of IDs of nominals, i.e., rels.

In turn, doins of the types of do classifiers and rels, by definitions, are classifiers as well while the kernel of the corresponders (cors), i.e., regularities (regs), are not. .

5.3.3. Complex doins of high degree layers of cesTh, i.e., mentals, induce incrementally growing up systems of classifiers and explicit rels between them.

In turn, higher layers of neurons nets are systems of classifiers of lower ones with rels implicitly represented, seemingly, by synaptic links.

5.3.4. ANN can, we assume, equally represent cesThregs, thus, any cors.

Positive expectations follow, particularly, from interpretation of conditional or not reflexes by Pavlov via cors.

For example, conditional reflex of secretion of saliva to food accompanied with lighting cause conditional reflex of the same secretion to the lighting without the food.

In other words, activation of neuron classifiers $\mathrm{Cl}$ of food accompanied with activation of classifiersCl' of lighting generates positive synaptic links of the causal type between those classifiers.

As a result, a time type casual rels "lighting causes food "between IDs of $\mathrm{Cl}$ and $\mathrm{Cl}$ ' is formed.

And lighting will activate that rels rising expectations for the food that, apparently, can be eliminated if regularly fails.

While rels "lighting cause food "is a do classifier it can be interpreted also as regs

ifthe appearance of lighting on the left indicates that food can be expected, thus, if lighting then food cors can be processed in reasoning and algorithms. 
5.3.5. Recalling, that amount of synaptic links in NN of any human concurs with the amount of particles in universe it is worth to assume that neurons with synaptic links between them provide an example of constructive implementation of rels and cors.

4. To resolve completely the eANN?cesTh open, yet, state, at least, the following questions.

5.4.1. Assuming ANN can equaly represent any cesTh classifier and cors whether they can represent cesTh algorithms, abstract classes and abstracts explicitly or not, and if explicitly then how the ANN have to be organized?

5.4.2.How human- computer communications including explanations and understanding of mss, prognostication of utilities and search strategies having proper cesTh models can be interpreted in ANN?

5.4.3. Whether the rels between IDs of nominals can be implemented in cesTh analogously to ones in NN, seemingly, realized by activation of synaptic links between target nominals?

5.4.4. Eventually, how mss identified by psychologists can be equally interpreted both in ANN and cesTh?

\section{Challenging Cellular Negentropicity}

6.1. Evolutionary cellularswere always fighting and competing for energetic resources because any environment they chose to reproduce themselves is physically restricted by energy that, inevitably, causes competition and fighting for the survival.

6.2. Mental power of humans lets them become a unique cellulars approaching the entire sources of energy of the Universe, thus, getting an opportunity to exempt fighting for the survival.

6.3. 1.Picks of mental power of humans in the scales of its effectiveness and efficiency include, particularly, the following ones.

6.3.2. Awesome coverage by classifiers of the entire types of realities of the Universe and enormously expansion of the amount of classifiers.

Classifications span from particles, black energy and matter to cosmic spaceships, from sequencing of genomes to gene engineering, from computers to global networks and their controllers.

An estimate of the amount of human classifiers can be the number of about 300, 000.00 units in English dictionaries.

6.3.3. Continuous rise of the abstractness and universality of classifiers.

6.3.4. Approaching the regularization of classifiers of any external or internal realities.

Starting from the grown up regularization, say plants and domestic animals, humans are intensively progressing in constructive regularization of mental doings, specially cognition, that exempting from cellar dependency enhances the dimensions of empowering.

6.3.5. Invention of organizations effectively integrating humans for common goals, particularly for the cognizing.

6.4. The above and other dimensions of mental power let question whether humans with unlimited access to the sources of energy can organize themselves into a type of negentropics that instead of fighting for energetic resources will prioritize their mental doings, say, cognizing of Universe, to become so knowledgeable that could, at least, serve their successful ongoing access to the energy and be ready to prevent possible troubles with it.

6. 5.1. An unconquered obstacle to organizations of humans, particularly, to ones with domination of cognition, is in their evolutionary genomic heritage to fight for the superiority and subsequent possible aggressiveness, greediness, evilness, cruelness resulting in evasion of members of organizations from routine obligations or their erosion by corruption. 
To avoid routine work and servicing for humans we could assume that with the growth of intelligence of machines they would be able to take that burden while humans could concentrate on creative work and cognition.

Unfortunately, those options rise new serious problems.

With passing services to machines humans have to delegate them certain control that inevitably will result in malicious usage of that control [34].

Then, deepening of cognition assumes effective cognizing organizations that again meets the obstacles with human genomic nature while the transmission of cognition to the machines challenges the further being of humans, at least, as a type of cellulars.

6.5.2.The questions arise whether humans being enough mentally empowered can transit to new types ofnegentropics free from drastic aspects of heritage of humans and able to preserve the attained advances in serving energy.

And whether those negentropicss with dominating cognition can be classified as a new type of descents of humans consistent with their further being or as ones conquering with them for the unconditional priority.

Those fundamental, critically important questions stay open yet and hopefully can be resolved with deepening cognition of ourselves and universe.

6. 6.1. Thus, acknowledging nowadays intractability of the problem DDPEN of doingsfor quasi eternal, durable preservation of energized negentropicityin the universe let's focuson the studying of mental doings of humans that to certain extent are invariant wrtall the types of negentropics.

Namely, let's focus on the problem of identification of mental doings of negentropics necessary for all of them for preserving energized negentropicity from the challenges of environments.

In other words, for the class ENs of energized negentropics including not only humans but all negentropics having sufficient technologies to access to quasi eternal sources of energy we can state the problem IMDE PEN of identification of mental doings necessary for all negentropics of ENs in preservation of energized negentropicity (PEN) and effectiveness of those doings in PEN.

6.6.2. To analyze mental doings of negentropics in EN we, apparently, refer to human ones as the base.

Namely, so far we have presented the modified specification of certain constructively regularized models (CRM) of mss, mentals, introduced in [47] for the class MS of mss, i.e.,the class MS representing entire mss, that, we assume, can be adequate CRM for human mental doers and systems, mdoers and mss.

Mdoers and mss, have, we assume, at least, IDs and can be processed consciously, particularly, for explaining and understanding doings/doers in communications.

Construction of mentals refer only to the existence of sensors, effectors and mental controllers of humans as well as, in general, to their goal oriented doing without addressing the peculiarities of goals of any type of negentropics.

Thus, accepting that sensors, effectors and mental controllers are inevitable for any negentropic, we assume that CRM for humans, mentals, can be considered as CRM for all negentropics including ones of the class EN.

6.6.3. The scales of effectiveness of mental doings wrt PEN are diversified and include the ones of coverage of classified realities, abstractness, universality and regularization of classifiers.

Focusing on the scales of constructive regularization of mental doings, we aim to approach to solving the problem CRMD PEN.

And as step to solving CRMD, it is reasonable to study cognitive doings, or constructive regularization ofcognition, as the problem CRC PEN as well as sub problems of CRC including regularization of mss representing inductive and deductive inferences, acquisition, accumulation, enhancement of effectiveness, controllers and communications. 
In the frame of the above assumptions, we are going to continue to advance in adequacy of mentals for the constituents of cognizers started in [47] as well as continue to develop models of CRC in [36-46]

6.7. To advance in CSC PEN we need to question the range of mental doings of humans inevitable for any cognizing negentropic of EN.

Certain mental doings of humans are the subject of studying of cognition in AI in certain pragmatic human-machine contents but whether they cover the scope of ones inevitable for negentropics of EN with cognizers estranged from celulars.

Whether motivation, will, intuition, consciousness, other dimensions of personality of humans have to be necessarily regularized?

Mental patterns are consequent to the dimensions of doing of humans and include, particularly, the following types.

1. Cognitive

2.1.Everyday life : adaptation to self-care, health and safety, social interactions and transactions at home, school and work, memorization of basic instructions, personal data (name, address) and important interests, goals setting and problem solving, judgments as well as doing integrated, i.e., setting goals, making decisions then judgment of the consequences

2.2. Highest

3. Communitive

4. Humanistic and ethical

Thus, we need to mirror mental patterns of human mss then target the ones inevitable for negentropics of EN.

\section{Conclusions}

7.1. We continue studying of adequate constructive models of mental systems.

First, we present the modified specification of the models of mss, mentals, introduced in [47], refine systemic classifiers and constructive regularization of classifiers.

Then, question the ways of proving that mentals can be adequate constructive models of mss and analyze the ways to achieve consistency of performances of structural models of connectivity neuron nets, for example, artificial neuron nets, with purely functional models of mss, mentals.

Assuming that the kernel of effective cognition is universal for all types of negs, we assume that scenarios with dominating cognition are the most promising to resolve the challenges of evolvement of humans and are the most expected for the next stage of their being.

7.2. We argue that mss, apparently, have to be prioritized with respect to the necessities of the most expected scenarios of human development and positioned in corresponding scales.

Given expected scenario the patterns of mss have to be revealed all together approximating relevant to scenarios basic strata of human mss.

For that, we assume, the views, particularly, on the nature of cognition and mental healthiness by philosophers, psychiatrists and psychologists have to be recalled as well as the views on organizational consistency by personal managers and ones on the norms of humanistic and ethical behavior.

Finally, from prioritized for given scenarios mss have to be excluded mss with ad hoc adequate constructive models to focus the research on the rest of the targets. 


\section{References}

[1] R. Feynman, The Meaning of it All, Addison Wesley, Massachusetts, 1998.

[2] J. von Neuman, Theory of Self-reproducing Automata, University of Illinois Press, 1966.

[3] T. Winograd and F. Flores, Understanding Computers and Cognition. A new foundation for design Publishers, Huntington, NY, 1986.

[4] J. Flavell, The Developmental Psychology of Jean Piaget, D.VanNostrand Comp. Inc., Princeton, N.J., 1962.

[5] Z. Pylyshyn, "Seeing and Visualizing: It's Not What You Think”, An Essay On Vision and Visual Imagination, http://ruccs.rutgers.edu/faculty/pylyshyn.html, 2004.

[6] E. Shrodinger, Mind and Matter, Cambridge, 1956.

[7] J. Mandler, The Foundations of Mind: Origins of Conceptual Thought, Oxford Univ. Press, 2004.

[8] A.Turing, Computing Machinery and Intelligence, Mind 49, 1950, [Reprinted in Minds and machines. A. Anderson (ed.), Engelwood Cliffs NJ, Prentice Hall, 1964].

[9] N. Chomsky, Aspects of the Theory of Syntax, MIT Press, 1965.

[10] J. Pitrat, "Consciousness and conscience, in artificial beings: the conscience of a conscious machine”, ISTE, London, UK, 2009.

[11] K Fu, Syntactic Methods in Pattern Recognition, London, 1974.

[12] E. Zermelo, "Ubereineanwendung der mengenlehre auf die theorie des Shachspiels", Proceedings of the fifth International Conference of Mathematicians, Cambridge University Press, 1912, pp. 501-504,

[13] J. Searle, “Is the brain’s mind a computer program?,Scientific American, vol. 262, pp. 26-31, 1990.

[14] D. Roy, "Grounding language in the world: signs, schemas, and meaning cognitive machines group”, The Media Laboratory, MIT http://www.media.mit.edu/cogmac / projects. html, pp. 127, 2005.

[15] C. Shannon, "Programming a computer for playing chess”, Philosophical Magazine Ser.7, vol. 41, 1950.

[16] Ch. Brutyan, I. Zaslavski, and L. Mkrtchyan, "On methods of automated synthesis of positional strategies in games”, ProblemiKibernetiki, Moscow, vol. 19, pp. 41-75, 1967.

[17] M. Botvinnik, "About solving approximate problems”, S. Radio, Moscow, (in Russian), 1979.

[18] M. Botvinnik, "Computers in chess: solving inexact search problems", Springer Series in Symbolic Computation, with Appendixes, Springer-Verlag:, New York, vol. ?, pp. ??, 1984.

[19] A. Elo, The Rating of Chess Players, Past and Present, London, 1978.

[20] J. Laird,The Soar Cognitive Architecture, MIT Press, England, 2012.

[21] (2013) [Online]. Available: UNDL Foundation, www.unlweb.net.

[22] “UNL Specifications", UNL Center of UNDL Foundation, 2005.

[23] F. Gobet, “Chunking mechanisms in human learning”, Trends in Cognitive Sciences, vol. 5, pp. 236-243, 2001.

[24] G. Atkinson, Chess and Machine Intuition, Ablex Publishing Corporation, New Jersey, 1993.

[25] J. Furnkranz, "Machine Learning in Games: A Survey in "Machines that Learn to Play Games”, Nova Sci., 2001.

[26] J. Moon, Topics on Tournaments, N.Y., Holt, and Winston, 1968.

[27] R. Benergi, Theory of Problem Solving,Mir, Moscow, 1972.

[28] R. Penrose, The Emperor's New Mind, Oxford University Press, 1999.

[29] A. Markov and N. Nagorni, Theory of Algorifms, Nauka, Moscow, 1984.

[30] A. Maltzev, Algorithms and Recursive Functions, Nauka, Moscow, 1965. 
[31] H. Marandjian, "A method of synthesis of programs of numeric functions", Mathematical Problems of Cybernetics and Computer, Yerevan,vol. 26, pp. 5-13, 1986.

[32] F. Harary, Graph Theory, Addison-Wesley, 1969.

[33] Perlov D. VilenkinA.Cosmology for the Curious. Springer, 2017

[34] Harari Y.N. Homo Deus: A Brief History of Tomorrow. Harper-Collins, 2017

[35] Helena Blavatsky: Between Light and Darkness. Жизнь замечательных людей (inRussian). Moscow: Молодаягвардия, 2010

[36] E.Pogossian and A. Martirosian . "Learning”, Reference book on Intellectual Systems. Radio i Svjas Publishing Company, Moscow, (in Russian), vol. 2, pp. 206-231, 1990.

[37] E. Pogossian, “On modeling cognition”, Computer Science and Information Technologies (CSIT11), Yerevan, 2011, pp 194-198, 2011.

[38] E. Pogossian, "Specifying personalized expertise. International Association for Development of the Information Society (IADIS)", International Conference Cognition and Exploratory Learning in Digital Age(CELDA 2006), Barcelona, Spain, 2006, pp. 151-159.

[39] E. Pogossian, V. Vahradyan, and A. Grigoryan, "On competing agents consistent with expert knowledge”, Lecture Notes in Computer Science, AIS-ADM-07: The International Workshop on Autonomous Intelligent Systems - Agents and Data Mining, St. Petersburg, Russia, June 67, 2007, pp. 229-241.

[40] E. Pogossian, "On measures of performance of functions of human mind”, 6th International Conference in Computer Science and Information Technologies, CSIT2007, Yerevan, 2007, pp. 149-154.

[41] E. Pogossian, "On a transparent presentation of written English syntax", 5th Intern. Cognitive Linguistics Conference, VrijeUniversiteit, Amsterdam, July 1996, pp. 209-214.

[42] E. Pogossian, “Adaptation of combinatorial algorithms”, Academy of Sci. of Armenia, (in Russian), p. 293, Yerevan, 1983.

[43] E. Pogossian, "Effectiveness enhancing knowledge based strategies for SSRGT class of defense problems", NATO ASI 2011 Prediction and Recognition of Piracy Efforts Using Collaborative Human-Centric Information Systems, Salamanca, Spain, 2011, pp. 16.

[44] Z. Naghashyan and E. Pogossian, "Developing Java software for representation, acquisition and management of strategy knowledge", Mathematical Problems of Computer Sciences, Proc. of IIAP, Yerevan, pp.187-195,2010,

[45] K. Khachatryan and S. Grigoryan, "Java programs for matching situations to the meanings of SSRGT games”, Proceedings of SEUA Annual conference, Yerevan, Armenia, 2013, pp. 135-141.

[46] S. Grigoryan "Research and development of algorithms and programs of knowledge acquisition and their effective application to resistance problems", $P h D, p 111$, Yerevan, Armenia, 2016.

[47] E. Pogossian, "Towards adequate constructive models of mental systems", 12th International Conference in Computer Science and Information Technologies, CSIT2017, Yerevan, 2017, pp. 96-101,2017, as well as IEEE's Xplore electronic library and is available at the link :http://ieeexplore.ieee.org/xpl/mostRecentIssue.jsp?punumber=8307363

[48] E. Pogossian, S. Grigoryan and N. Hakobyan, "On Systemic Classification and Machine Learning”, 12th International Conference in Computer Science and Information Technologies, CSIT2017, Yerevan, 2017,pp.102-107.

[49] S. Grigoryan, N. Hakobyan and H. Vrtanesyan, “Object -oriented Modeling of Systemic Classifiers and Matching to Classifiers", Mathematical Problems of Computer Sciences, Proc. of IIAP, Yerevan, pp.102-107,2018.

Submitted 22.09.2017, accepted 12.02.2018. 


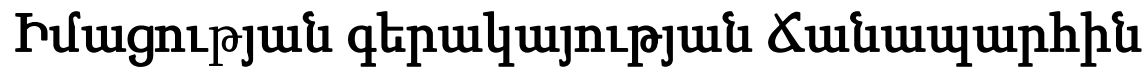

\author{
Ł. Tnпnujuiq
}

\section{Uự̧nน̣nıน}

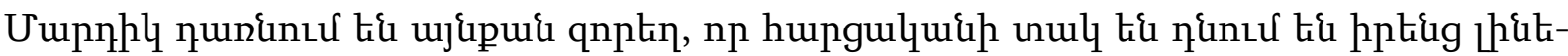

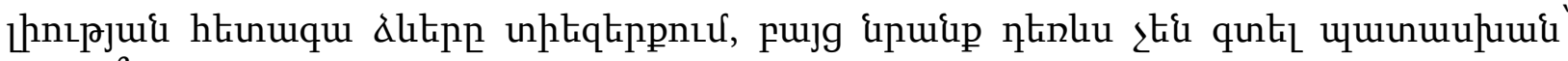

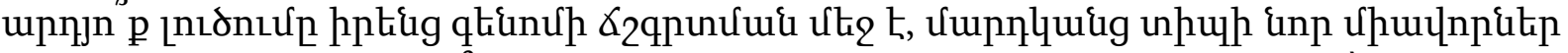

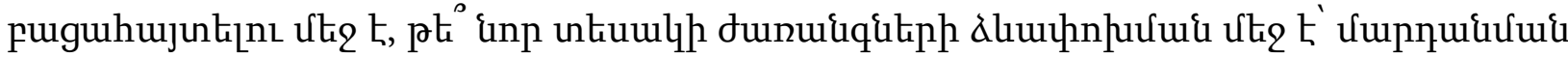

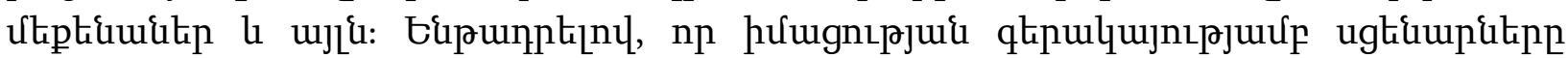

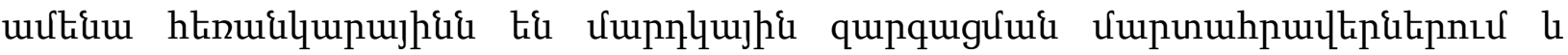

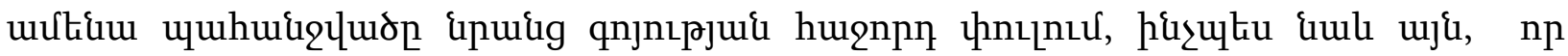

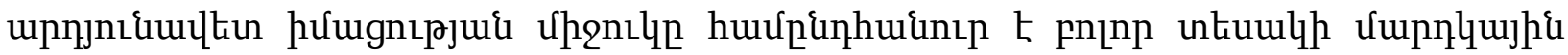

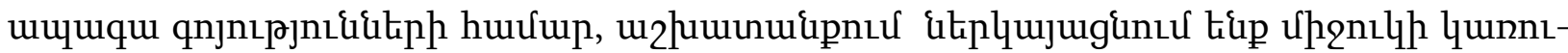

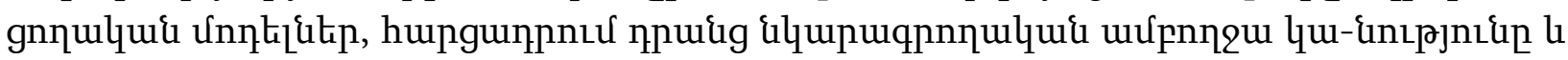

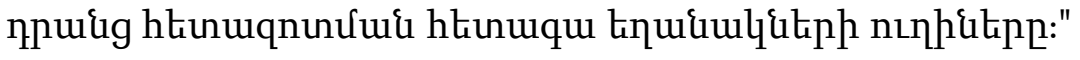

\section{К доминированию познания}

\section{Э. Погосян}

\begin{abstract}
Аннотация
Знания и способности человека приближают его к возможности энергетически надежного сохранения своего существования, но параллельно и к возможности полного уничтожения. Сила людей существенно определяется эффективностью их организаций, одновременно, их слабость обусловлена несовершенством этих же организаций, а также, геномным наследием человека.

Знания человека становятся достаточными, чтобы подвергнуть сомнению устойчивость клеточной организации бытия во вселенной , но пока не дают ответа, находятся ли решения в исправлении генома человека, обнаружении новых типов организации людей или в переходе к новому типу человекомашинного развития.

Мы считаем, что сценарии с доминирующим познанием являются наиболее перспективными для ответа на вышеуказанные проблемы и являются наиболее ожидаемыми для следующего этапа развития человека. Полагая, что ядро эффективного познания является универсальным для всех типов негэнтропного бытия, мы сосредотачиваем исследования на извлечении и изучении этого ядра, в частности, предлагаем конструктивные модели ядра, обсуждаем полноту и вопросы дальнейших исследований.
\end{abstract}

